Itinéraires Itinéraires

Littérature, textes, cultures

2018-2 et $3 \mid 2019$

Les imaginaires de la traduction

\title{
Fiodor Dostoïevski traducteur de Balzac : vers la révélation d'un futur talent littéraire
}

Fyodor Dostoevsky's Translation of Balzac: The Revelation for a Future Literary Talent

Lilia Androsenko

\section{OpenEdition}

\section{Journals}

Édition électronique

URL : http://journals.openedition.org/itineraires/4582

DOI : 10.4000/itineraires.4582

ISSN : 2427-920X

Éditeur

Pléiade

Référence électronique

Lilia Androsenko, « Fiodor Dostoïevski traducteur de Balzac : vers la révélation d'un futur talent littéraire », Itinéraires [En ligne], 2018-2 et 3 | 2019, mis en ligne le 20 février 2019, consulté le 18 juin 2019. URL : http://journals.openedition.org/itineraires/4582 ; DOI : 10.4000/itineraires.4582

Ce document a été généré automatiquement le 18 juin 2019.

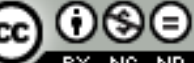

Itinéraires est mis à disposition selon les termes de la licence Creative Commons Attribution - Pas d'Utilisation Commerciale - Pas de Modification 4.0 International. 


\section{Fiodor Dostoïevski traducteur de Balzac : vers la révélation d'un futur talent littéraire}

Fyodor Dostoevsky's Translation of Balzac: The Revelation for a Future Literary Talent

Lilia Androsenko

«Balzac reste longtemps pour Dostoïevski ce qu'avait été Virgile pour Dante, lo maestro e l'autore - le maître qui l'associa au secret de son art et le guide le plus sûr dans tous les passages ténébreux

et dangereux. »

Leonid Grossman, Poètika Dostoevskogo, 1925.

\section{Introduction}

1 Grand admirateur de l'œuvre balzacienne, Fiodor Dostoïevski se nourrit dès son adolescence des thèmes et des idées de La Comédie humaine, ce qui lui fait avouer, dans sa lettre du 9 août 1838 adressée à son frère Mikhaïl : «Balzac est géant ! Ses personnages sont l'œuvre du génie universel! » (Dostoïevski 1998: 165). Plus tard, en 1843, le court séjour du romancier français à Saint-Pétersbourg ne fait qu'accroître l'enthousiasme du jeune Russe pour ce grand auteur et son œuvre, le déterminant à consacrer sa première publication rémunérée à la traduction d'Eugénie Grandet, roman qui aura une influence notable sur son œuvre romanesque.

2 Ayant achevé le travail en quelques semaines seulement, Dostoïevski le qualifie, dans une autre lettre à son frère, de « véritable merveille » et «sans pareille » (Dostoïevski 1998 : 212), malgré les transformations importantes qu'il fait subir au roman français. Publiée en juillet 1844 dans la revue saint-pétersbourgeoise Répertoire et Panthéon ${ }^{1}$ sous une forme 
anonyme, la traduction dostoïevskienne passe inaperçue aux yeux de la presse de l'époque, mais sert néanmoins de point de départ à la carrière du futur romancier.

3 Si Dostoïevski sent, dès son plus jeune âge, la parenté de l'œuvre de Balzac avec sa propre vision de la littérature, c'est ce travail sur Eugénie Grandet qui développe chez lui une véritable "pensée de la littérature, une pensée du langage » (Meschonnic 1999: 20), qui se cristallisera dans ses futurs écrits. Sans trahir l'intrigue du roman, le jeune homme âgé de vingt-deux ans se soucie peu du « contenu inessentiel » (Benjamin [1923] 1971 : 282) de l'original, son but étant de faire vivre à son lecteur toute la palette des émotions que luimême avait éprouvées lors de sa lecture du roman. Guidé par son instinct de poète ${ }^{2}$, Dostoïevski abandonne l'exactitude et la fidélité ${ }^{3}$ au profit d'une nouvelle esthétique de l'original qui sera fondée sur l'impression, la sensation et la perception subjective. Cette approche poétique et imagée évoque d'ores et déjà les conditions nécessaires à la création d'une œuvre romanesque, avancées par lui-même vers la fin de sa vie :

Pour écrire un roman, il faut avant tout se procurer une ou quelques fortes impressions, que le cœur de l'auteur a réellement vécues. En cela consiste la tâche du poète. De cette impression se développe un sujet, un plan, un tout organique. Ici, c'est déjà l'affaire d'un peintre, bien que le peintre et le poète s'entraident dans les deux cas. (Dostoïevski, cité dans Nečaeva 1979:3)

4 À la fois traducteur et artiste, Dostoïevski fait revivre Eugénie Grandet dans une perspective originale et particulièrement féconde, faisant de l'œuvre balzacienne un atelier de réflexion et de création littéraire.

La démarche de découverte et de détermination du champ des possibles du traducteur à travers son premier travail de traduction littéraire nous amène naturellement à nous intéresser au phénomène de la traduction productive - initiatrice d'un ouvrage littéraire indépendant lié au texte traduit par une relation d'hypertextualité ou d'intertextualité -, qui se déploiera, dans la version russe d'Eugénie Grandet, à la fois au niveau thématique, en tant que transposition et transcendance des personnages du roman, et stylistique, en tant que contribution au développement d'un style personnel, révélant chez le traducteur le futur talent artistique de la littérature.

\section{La pensée d'une traduction ethnocentrique ${ }^{4}$}

6 À l'instar des traducteurs russes des années 1840, Dostoïevski se préoccupe avant tout de la pensée poétique et romanesque du texte, qu'il met en œuvre en tirant parti du potentiel expressif de la langue russe ${ }^{5}$. C'est ainsi que, libérée des contraintes imposées par la fidélité à la lettre, la traduction se décharge de la complexité du vocabulaire balzacien, tout en privilégiant l'essentiel au détriment du secondaire et ne gardant que l'idée générale du texte initial.

7 Pourtant, l'abondance des realia, propres au système lexico-syntaxique de la langue russe et à l'imaginaire culturel du pays, s'explique également par des choix personnels de Dostoïevski en termes de vocabulaire et de réorganisation structurelle du texte source, ce qui nous pousse à explorer le caractère intertextuel de leur emploi à l'échelle de l'œuvre romanesque du futur romancier russe.

8 Tout d'abord, le traducteur utilise à profusion les appellations officielles de la Russie monarchique du XIX ${ }^{e}$ siècle: parmi les trois traductions synonymiques des madame et monsieur français (alternance de barin-barynja-baryšnja ${ }^{6}$, de sudar'-sudarynja et de gospodin- 
gospoža), Dostoïevski témoigne d'une préférence claire pour le soudar', de même qu'il recourt avec insistance au phénomène de slovoyers (le -s final de respect, de politesse ou de rabaissement devant l'interlocuteur qui clôture la réplique) : «Da-s , slušaju [Oui, monsieur, j'écoute]» (Dostoevskij 1844: 51)7 . Dans leur rôle d'équivalents ethnolinguistiques, ces formules de politesse annoncent d'emblée le principe dialogique de la future œuvre dostoïevskienne développé par Mikhaïl Bakhtine ${ }^{8}$, tandis que le slovoyers deviendra, chez le romancier russe, une marque de fragilité, de vulnérabilité psychologique du protagoniste devant l'autre, comme c'est le cas dans Notes de sous-sol où le narrateur a recours au -s final plus de vingt-cinq fois.

9 Le goût de Dostoïevski pour le langage familier, le registre populaire de la langue russe, pour les expressions figées et le langage imagé - ce qui lui sera souvent reproché par ses contemporains - apparait d'ores et déjà dans sa traduction d'Eugénie Grandet. Dans un souci d'authenticité, le traducteur recourt aux particules-postfixes, ces courts mots invariables qui, dans les langues dites flexionnelles, indiquent les mouvements de pensée, mettant en relief la sémantique d'un mot auquel ils se réfèrent ${ }^{9}$. Utilisés pour la plupart comme équivalents de donc, allons (-ez), ben, les -ka, -že, -to russes «desserrent » la narration, tout en mettant l'accent sur l'oralité du discours. La phrase impérative de la servante Nanon : « Demandez-lui du beurre [...]. Tenez, le voilà qui descend pour voir aux provisions...» (Balzac 1834: 129) s'enrichit, chez Dostoïevski, de cinq particules et de

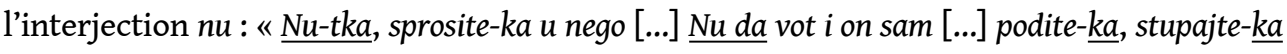
knemu!... » (1844: t. 6, 431).

Les passages narratifs de la traduction abondent en substantifs et épithètes aux suffixes diminutifs qui - propres notamment aux premiers écrits de Dostoïevski - apportent une affection particulière aux passages en question. Ainsi, «une fille propre, pimpante de jeunesse, au blanc fichu, aux bras rouges » (Balzac 1834: 26) se dote, chez le traducteur, de trois adjectifs et deux substantifs en forme diminutive: "une fille proprement habillée, toute jolie [horošen'kaja], toute jeune [moloden'kaja], au petit blanc fichu [kosynočke], aux petits bras dodus [s puhlen'kimi krasnymi ručkami]» (1844: t. 6, 388), alors que l'ameublement et le décor de la maison Grandet semble rapetisser sous le poids du même procédé : les "chaises à bois jaune», le «maigre tapis» de Balzac (1834:115) se transforment en « petites chaises jaunes [z̈Ëlten'kie stul'iki] » et « petit tapis étroit avec des fleurs quelconques [uzen'kij kovËr s kakimi-to cvetočkami] ${ }^{10}$ » (Dostoevskij 1844 : 425).

11 L'envie de naturaliser le texte de départ, de lui donner une orientation russophile amène le traducteur à enrichir le contenu stylistiquement neutre de l'original ou bien dénué de sens pour un lecteur russophone, d'expressions populaires. Dans « Eugénie, jaune comme un coing » (Balzac 1834: 106), Dostoïevski remplace le dernier mot par « cire », croyant qu'il reproduira mieux le teint jaunâtre de la jeune femme dans l'imaginaire du lecteur. La réplique du père Grandet: "Je travaille à mon âge comme un jeune compagnon " (Balzac 1834: 62), est traduite avec deux éléments comparatifs traditionnels, d'une part, « comme un bœuf» [kak byk], et d'autre part, « comme le dernier batrak» [kak poslednij batrak] (Dostoevskij 1844 : t. 6, 423), les batraks étant, dans la Russie d'avant la Révolution, des ouvriers agricoles de rang social peu élevé. Enfin, pour traduire: "Je le croyais sourd» (Balzac 1834 : 284), Dostoïevski introduit la comparaison bien connue des Russes : «J'ai cru qu'il était sourd comme un tétras [teterev]» (t. 7, 84), alors que la phrase «Il dort comme s'il était le roi de la terre » de Balzac $(1834: 143)$ est remplacée par l'expression idiomatique «Il dort d'un sommeil de mort [mërtvym snom] » (Dostoevskij : t. 6, 437), c'està-dire d'un sommeil très profond. 

la suppression des mots étrangers non français ou encore par l'introduction d'épithètes et de substantifs folkloriques. Ainsi, «son dégrisement alla rinforzando » (Balzac 1834 : 111), ou encore « un crescendo d'étonnement » (Balzac 1834: 82) se voient remplacées par «il crut se retrouver dans un poulailler [čto popal v kurjatnik] » (Dostoevskij : t. 6, 424) et « un étonnement croissant [vozrastavšee udivlenie]» (Dostoevskij: t. 6, 412). L'épithète folklorique bogatyrskij ${ }^{11}$ (Dostoevskij: t. 6, 399) vient qualifier la grande taille et la remarquable endurance de la Grande Nanon, tandis que le substantif familier kubyška (récipient en argile au col étroit, une sorte de jarre, par ext. endroit où l'on garde l'argent, une tirelire) serait plus à même, selon Dostoïevski, d'évoquer chez le lecteur l'imaginaire de l'argent et de la cupidité propre au père Grandet. Nous ne citerons ici qu'un seul exemple d'utilisation de ce dernier par Dostoïevski : la description du cabinet du vieil avare, où « ...quelque cachette avait été très habilement pratiquée » (Balzac 1834 : 113), est librement traduite par : «Ici, était probablement cachée sa chère kubyška » [«Zdes'-to, verojatno, byla zaprjatana zavetnaja kubyška »] (Dostoevskij 1844 : t. 6, 424).

La préférence que donne le traducteur à sa langue maternelle se révèle donc non seulement un principe traductologique de l'époque, mais aussi un parti pris personnel de Dostoïevski, qui sera qualifié par Nikolaj Berdjaev $(2016$ : 317) « de génie profondément russe, le plus russe de tous nos grands écrivains ».

\section{Parti pris sensible : les obsessions du père Grandet russe}

Prisonnier « d'un style théâtral qu'il maniait alors » (Catteau 1978 : 26), Dostoïevski ne se contente que rarement de garder la syntaxe initiale du roman, préférant la transformer en fonction de la touche émotionnelle qu'il désire communiquer au texte cible. Si la phrase de Balzac lui paraît trop pesante et excessivement développée, il la coupe et en synthétise le contenu. Si, en revanche, elle mériterait, selon lui, d'être explicitée davantage, il en « renforce et précise les impressions » (Nečaeva 1979: 116) par des mises en relief lexicales et syntaxiques.

Disant consciemment les personnages en registres stylistiques et psychologiques différents, Dostoïevski retravaille leur évolution personnelle dans les moindres détails en fonction de son sentiment vis-à-vis de ces derniers. Chaque portrait étant stylistiquement affecté, Eugénie et sa mère apparaissent sous des traits à la fois romantiques et tragiques, alors que le père Grandet russe devient l'incarnation même de l'avarice, du cynisme et de la brutalité.

16 Le traducteur russe repense la syntaxe, le lexique et la ponctuation du texte français de manière à grossir les défauts du tonnelier russe - rusé, tyrannique et obnubilé par sa fortune -, à mener à l'extrême son obsession maladive pour l'or tout en réduisant sa vie à l'argent.

17 Lorsque le père Grandet se réveille "de ses méditations » (Balzac 1834: 177), son homologue russe "se secoue de ses "zéros", de ses chiffres, de ses totaux et ses calculs" (Dostoevskij 1844 : t. 6, 452). Alors que, chez Balzac, il vient «choyer, caresser, couver, cuver, cercler son or » (1834: 113), chez Dostoïevski, il « ouvre sa koubychka, compte à nouveau son or, le regarde avec avidité, des heures entières, le pèse sur la balance, dans ses mains, embrasse son trésor avec amour, avec plaisir» (1844: t. 6, 424). L'amour 
excessif du vieil homme pour l'argent est également mis en relief par la pauvreté et la vulgarité de l'ameublement de sa maison ainsi qu'à travers les paroles de son entourage, dont madame des Grassins qui reproche au tonnelier d'être « la vieille mouche du coche [qui] n'a en tête que le commerce, des gains, des calculs [torgovlja, baryši, rasčËty] " (Dostoevskij $1844:$ t. 6, 417), pour « un grigou qui ne pense qu'à ses provins » de Balzac (1834: 96). Sans citer tous les exemples, il est clair que la méthode du traducteur - qui consiste à prolonger le champ lexical de l'argent et du commerce - sert à repenser le contenu et l'agencement syntaxique de la phrase balzacienne, à mettre l'accent sur une idée bien précise par l'entassement de synonymes : par conséquent, la longueur de la phrase traduite dépasse souvent celle du texte original.

Jouant sur des contrastes, Dostoïevski ajoute à plusieurs répliques du cruel Grandet des diminutifs à valeur affective ${ }^{12}$ qui, censés délimiter les champs d'affection du personnage, mettent en lumière son amour pour l'argent (denjužki) et Eugénie, qui en est l'unique héritière (plutovočka, miločka, dušečka, dočečka, golubuška, etc.). Le suremploi des diminutifs par Grandet joue sûrement une double fonction dans la traduction : « humaniser » le dur tonnelier tout en jouant sur la dichotomie entre sa tendresse apparente et son insensibilité dissimulée ${ }^{13}$. Ainsi, la phrase brève et succincte du maitre de maison: "Tiens, vois, j'embrasse Eugénie, elle aime son cousin, elle l'épousera, si elle veut " (Balzac 1834: 320), se dote de quatre diminutifs dans la traduction de Dostoïevski: « Tiens, mon petit cœeur [dušečka], regarde, voilà que j'embrasse Eugénie, ma fille, ma chère petite fille [moju miluju dočečku]. La petite coquine [Plutovočka] aime le beau [krasavčika] Charles » $(1844:$ t. 7,101$)$. Pour la fifille de Balzac, le traducteur introduit un large champ synonymique d'apostrophes en forme de diminutifs qui visent toutes à clarifier la signification du substantif employé : « Mon amie, ma petite chérie [milušeka], ma petite fille [ dočečka]! Mon enfant! Ma petite douce [dušečka]!» (1844: t.7, 100), le traduisant quelquefois par le néologisme ma petite vie (žiznËnoček). Lors de la rédaction de Les Pauvres Gens (1844-1846), Dostoïevski aura recours aux mêmes diminutifs qui nourriront l'intertexte traductologique de son premier roman: celui-ci comptera dix-neuf occurrences de dušečka / dušen'ka (contre dix dans son Eugénie Grandet), deux de žiznenoček (contre un) et quarante-et-une occurrences de golubuška, contre trois dans la traduction.

Les suppressions textuelles que pratique en abondance le traducteur ne raccourcissent donc point l'original: de nombreux ajouts à valeur d'appréciation parsèment l'Eugénie Grandet russe de sorte à la couvrir de nouvelles images, faisant ressurgir quelque nouvelle nuance ou interprétation.

\section{Le tragique au féminin}

Profondément ancré dans la conception littéraire du romancier russe, le tragique fascine Fiodor Dostoïevski dès son plus jeune âge, ses premières expériences littéraires inachevées étant deux tragédies historiques, Marie Stuart et Boris Godounov. Plus tard, l'une des principales réalisations poétiques de la veine tragique de son œuvre deviendra la femme russe qui, de par son humilité et sa douceur, sera amenée à incarner le salut de l'homme.

La traduction d'Eugénie Grandet accorde déjà une place centrale au développement poético-lyrique des personnages féminins, par lesquels s'élabore une "poétique dramatique personnelle» (Alekseev 1921: 48) du futur romancier. Sa fascination pour 
Eugénie et sa mère qui, terrorisées par le maître de maison, ne s'abandonnent toutefois pas au désespoir - leur foi en Dieu leur donnant force et tranquillité face aux épreuves de la vie familiale - l'amène à retravailler la charge émotionnelle des épisodes où il est question des deux femmes.

Comparons les passages qui décrivent la mère d'Eugénie mourante :

Elle était frêle autant que les feuilles des arbres en automne; et les rayons du ciel la faisaient resplendir comme ces feuilles que le soleil traverse et dore. (Balzac 1834: 323)

Son existence ressemblait au vacillement d'une feuille jaune d'automne, frêle, desséchée, se tenant à peine sur une branche. Et tout comme le soleil, dont les rayons passent à travers les rares feuilles d'automne, les couvrant d'or et de pourpre, ainsi les rayons de la béatitude céleste et de la paix spirituelle faisaient resplendir le visage de la martyre mourante. (Dostoevskij 1844 : t. 7, 102)

Dans la traduction, le portrait de la femme mourante - retravaillé dans son aspect lexical et syntaxique - ouvre le roman original à une émotivité mélodramatique plus importante, à une rythmique et une expressivité stylistique nouvelles, à la fois pathétique, poétique et tragique. Paraphrase, inversion ${ }^{14}$, harmonisation de la disposition des qualificatifs (chaque substantif se dote d'une épithète), ajout des expressions figées du slavon d'église permettent au traducteur de mettre l'accent sur la dimension hautement spirituelle du moment et, d'autre part, d'accentuer davantage le parallèle métonymique entre la fragilité de la mère mourante et la mélancolie de la saison. La tendance dostoïevskienne à repenser la syntaxe, la ponctuation et le champ lexical de la phrase balzacienne se poursuit par une saturation de l'espace narratif de l'original qui déstabilise « le signe vers le rythme» (Meschonnic 1999: 36), et la placidité observatrice de Balzac vers une sensibilité baroque. Si le romancier français «invente un instrument d'une souplesse égale à ses analyses minutieuses» (Bertault 1968: 231), le jeune Russe y «apporte de l'exceptionnel", tout en alliant "selon le principe romantique le sublime et le grotesque " (Grossman 1925: 62). Guidé par son imagination créatrice, le traducteur " exalte de l'intérieur le style de Balzac", lui communiquant " une apparence nouvelle, extraordinaire » (Čičerin $1980: 159)$ : dans une envolée lyrique et de passion dramatique, Dostoïevski crée ici l'un des plus beaux passages de sa traduction.

À la croisée du lyrisme romantique et du pathos religieux, la poétique dostoïevskienne de la souffrance couvre l'image sublimée d'Eugénie Grandet de nouvelles nuances sémantiques absentes dans l'original. $\mathrm{Si}$, chez Balzac, son « amour [...] ne lui causait que des douleurs, mêlées de frêles espérances» (Balzac 1834: 338), dans la traduction, ce sentiment " pesait sur son cœur en le faisant languir de douleur et d'amertume; il brillait à peine dans l'avenir d'un fragile espoir lointain» (Dostoevskij 1844: t. 7, 109). Lorsque Balzac décrit l'apparence d'Eugénie - «Son visage est blanc, reposé, calme. Sa voix est douce, et recueillie » $(1834: 380)$ - Dostoïevski le traduit ainsi : « Les traits de son visage sont tendres, doux; il y a autour d'elle une couronne de dignité et de martyre» (1844 : t. 7, 124).

Intéressante est l'insistance avec laquelle le terme biblique krotost ${ }^{15}$ (douceur) et ses dérivés reviennent dans la traduction dostoïevskienne. Ainsi, "sa patience d'ange " (Balzac 1834: 323) se transforme en «sa douceur d'ange» (Dostoevskij 1844: t. 7, 103), « agneau sans tache» $(1834: 323)$ en «agneau doux» $(1844:$ t. 7,103$)$, alors que «des qualités morales qui venaient fleurir sur sa face " $(1834: 306)$ «luisent d'une douceur céleste » chez le traducteur russe $(1844:$ t. 7,94$)$. Vingt ans plus tard, cette même qualité prendra sa place, dans Crime et châtiment, à côté de la prostituée Sonia Marmeladova ${ }^{16}$ et 
de Lizaveta qui, tuée aussitôt par Raskolnikov, fera exclamer à ce dernier : «Lizaveta ! Sonia! pauvres douces créatures aux yeux doux! chères [...] Sonia, douce Sonia!» (Dostoïevski 1983: 329). Associée manifestement chez Dostoïevski au rôle de femmevictime et de femme-martyre, cette valeur chrétienne donnera également son nom à la nouvelle $\mathrm{La} \mathrm{Douce}^{17}$ (1878), où elle sublimera l'image de l'héroïne principale.

D'après le critique Vladimir Bienstock, Dostoïevski trouve dans Eugénie Grandet ce qu'il cherchait depuis longtemps, à savoir la « femme véritablement malheureuse » (Bienstock 1924 : 419), qu'il fera renaître à travers nombre de ses personnages féminins. Qualifié par la suite de "plus grand peintre des souffrances humaines et des maux de l'existence " (Evnin 1969 : 416), le traducteur jalonne, à travers le développement de la dimension poétique et tragique des héroïnes balzaciennes, le pathos de "la veine évangéliste" (Grossman 1925: 88) de ses futures œuvres romanesques, définies par le "pathétique, l'hyperbole et la brume mélodramatique » (Catteau $1978: 26$ ).

\section{Conclusion}

Hautement métapoétique et intertextuelle, la première traduction russe d'Eugénie Grandet coïncide avec le questionnement de son traducteur sur le potentiel expressif et créateur de l'art littéraire. Fasciné par ce roman psychologique et social qui lui «apprend ce que c'est que l'homme et la vie» (Nečaeva 1979: 104), Dostoïevski donne, à travers sa traduction, une réponse " personnelle et créatrice » (Eco 1965) au " problème d'identité et à celui de sa vocation » (Alekseev $1921: 52$ ).

Dès lors, la nature « riche, copieuse, opulente » (Bertault 1968 : 230) du texte de Balzac se voit transformée par la conception expérimentale créatrice de Dostoïevski : partant de la dimension analytique et observatrice de l'original, le traducteur développe l'intelligence de l'émotion des personnages, tout en faisant l'éloge de la sensibilité poétique d'une œuvre littéraire. Désirant traduire de façon à ce que l'on ne « sente » pas la traduction, de façon « à donner l'impression que c'est ce que l'auteur aurait écrit s'il avait écrit dans la langue traduisante » (Berman 1999 : 35), Fiodor Dostoïevski propose une œuvre littéraire hybride et polyphonique qui, à l'image de ses futurs écrits ${ }^{18}$, illustre la proximité intellectuelle et stylistique entre les deux romanciers, devenant ainsi pour le futur romancier russe un espace fécond de réalisation personnelle.

La silhouette ajourée, poreuse et palpitante du texte dostoïevskien évoque cette «spiritualité baroque» qui serait, selon Umberto Eco (1965: 20), «la première manifestation clairement exprimée de la culture et de la sensibilité moderne ». Envisagée non comme une restitution mais comme une transformation qui laisse « tomber le corps ${ }^{19}$ ", la première traduction russe d'Eugénie Grandet en appelle en effet à la modernité d'une pensée sur la poétique du traduire. Valoir non à la place de l'autre mais en ses lieu et place, tout en débordant «le binarisme auquel son espace fonctionnel, le vis-à-vis des langues, semblait devoir la soumettre » (Bernadet 2014 : 16), constitue le principe central de la première traduction russe d'Eugénie Grandet, qui dévoile les fondements de ce que sera l'écriture du grand romancier Fiodor Dostoïevski. 


\section{BIBLIOGRAPHIE}

Alekseev, Mihail, 1921, «O dramatieskih opytah Dostoevskogo », pod red. Leonida Grossmana, in Tvorcestvo Dostoevskogo [ « Des expériences dramatiques de Dostoïevski », dans L. Grossman (dir.), L'Euvre de Dostoïevski], Odessa, Vseukrainskoe gosudarstvennoe izdatel'stvo, p. 41-62.

Bakhtine, Mikhaïl, 1998 [1929], Problèmes de la poétique de Dostoïevski, trad. de Guy Verret, Lausanne, L'Âge d'homme.

Benjamin, Walter, [1923] 1971, La Tâche du traducteur, trad. Maurice de Gandillac, Paris, Denoël. Berdjaev, Nikolaj, [1923] 2016, Mirosozercanie Dostoevskogo [La Conception du monde de Dostoïevski], Moscou, Izdatel'stvo « È ».

Berman, Antoine, [1991] 1999, La Traduction et la Lettre ou l'Auberge du lointain, Paris, Seuil, coll. « Ordre Philos ».

Bernadet, Arnaud et Paien de La Garanderie, Philippe, 2014, Traduire-écrire : cultures, poétiques, anthropologie, Paris, ENS Éditions.

Bertault, Philippe, 1968, Balzac, Paris, Hatier, coll. « Connaissance des Lettres ».

Bienstock, Wladimir, $1^{\mathrm{er}}$ décembre 1924, «Balzac et Dostoïevski », Mercure de France, $\mathrm{n}^{\circ}$ 635, p. 419-426.

Catteau, Jacques, 1978, La Création littéraire chez Dostoïevski, Paris, Institut d'études slaves.

Čičerin, Alexej, [1973] 1980, Ritm obraza [Le Rythme de l'image], Moscou, Sovetskij pisatel.

Derrida, Jacques, 1967, L'Écriture et la Différence, Paris, Seuil.

Dostoïevski, Fiodor, [1832-1864] 1998, Correspondance. Tome 1, Paris, Bartillat.

Dostoïevski, Fiodor, [1866] 1983, Crime et châtiment, Paris, Gallimard.

Eco, Umberto, [1962] 1965, L'Euvre ouverte, trad. Chantal Roux de Bézieux avec le concours d'André Boucourechliev, Paris, Seuil.

Evnin, FËdor, 1969, « Realizm Dostoevskogo », dans Problemy tipologii russkogo realizma, pod red. N.L. Stepanova i U.R. Fohta [« Le réalisme de Dostoïevski », dans N. L. Stepanov, U. R. Foht (dir.), Problèmes de typologie du réalisme russe], Moscou, Nauka, p. 409-453.

Fainštein, Mihail, 1989, Pisatel'nicy pouškinskoj pory. Istoričesko-literaturnye očerki [Les Femmes écrivains du temps de Pouchkine], Léningrad, Houdožestvennaâ literatura.

Grossman, Leonid, 1925, Poètika Dostoevskogo [La Poétique de Dostoïevski], Moscou, GAHN, coll. « Istoria i teoria iskusstv ».

Meschonnic, Henri, 1999, Poétique du traduire I, Paris, Verdier poche.

Nečaeva, Vera, 1979, Rannij Dostoevskij. 1821-1849 [Le Jeune Dostoïevski. 1821-1849], Moskva, Nauka.

Payevskaja, Anastasyja et Dančenko, Vladimir, 1965, Onoré de Balzac. Bibliografija russkih perevodov i kritičeskoj literatury na russkom jazyke. 1830-1964 [Honoré de Balzac. La bibliographie des traductions et des ouvrages de critique en langue russe. 1830-1964], Moscou, Kniga. 
Pospelov, Gennadij, 1928, Eugénie Grandet Balzaka v perevode Dostoevskogo [Eugénie Grandet de Balzac dans la traduction de Dostoïevski], Moscou, Ranion.

Tučina, Maria, 2010, «Barokko F. M. Dostoevskogo kak poètologičeskaja problema » [« Le baroque de F. M. Dostoïevski comme problème poétologique »], dans K. E. Stajn et D. I. Petrenko (dir.), Metapoètika, $\mathrm{n}^{\circ}$ 2/1, Stavropol, p. 158-164.

\section{Corpus}

Balzac, Honoré de, 1834, Eugénie Grandet, Paris, Charles-Béchet.

Balzac, Honoré de, [1834] 1844, Eugénie Grandet, trad. FËdor Dostoevskij, dans Repertuar et Panteon inostrannyh teatrov [Répertoire et Panthéon des théâtres étrangers], Saint-Petersbourg, 1844, t. 6, kniga 6, p. 386-437 ; t. 7, kniga 7, p. 4-125 [le nom du traducteur n'est pas mentionné].

\section{NOTES}

1. Dans la présente étude, nous nous référons à cette première édition de la traduction dostoïevskienne. Nous utilisons la translittération des slavistes pour les références aux ouvrages russes, les noms (à l'exception du nom de Dostoïevski dans le corps du texte) et les citations extraites de ces derniers et traduites par l'auteure de l'article.

2. " Poète » est à prendre ici au sens grec du terme, c'est-à-dire, en tant que créateur, inventeur de nouvelles formes expressives. Dans l'Antiquité grecque, toute expression littéraire est qualifiée de poétique.

3. Selon Henri Meschonnic, ces deux notions nuisent au travail du traduire car elles «pensent étreindre le texte, et n'embrassent qu'un énoncé » (Meschonnic 1999 : 31).

4. Contraire d'exocentrisme. Ce terme est proposé par Antoine Berman dans La Traduction et la lettre ou l'Auberge du lointain, où il étudie les tendances de rapprochement (ethno-) et d'éloignement (exo-) de la langue et culture d'origine.

5. D'après les spécialistes de l'histoire des traductions de Balzac aux $\mathrm{XIX}^{\mathrm{e}}-\mathrm{xx}$ e siècles, « le lecteur des années 1830-1840 faisait malheureusement la connaissance de Balzac au travers de mauvaises traductions" car la majorité des traducteurs de l'époque «ne voyaient leur rôle que dans la transmission de l'intrigue, parfois même de façon approximative, sans jamais se soucier des particularités du style ou de la langue de l'écrivain » (Paevskaja et Dančenko $1965: 35$ ).

6. Dostoevskij 1844 :t. 6, 415, 426, 433, 438 ; t. 7, 75, 80, 83. Les renvois à la traduction se font avec le nom russe translittéré de Dostoïevski.

7. On trouve dans l'œuvre respectivement 52 et 57 occurrences de l'appellation sudar'/sudarynja (du russe gosudar', messire), tandis qu'on n'en compte que que 9 et 3 pour baryn. Appelée « slovoyers ", la forme abrégée de sudar' sert à marquer le respect et, lorsqu'elle est utilisée à l'excès, le rabaissement devant son interlocuteur.

8. Voir à ce propos Mikhaïl Bakhtine ([1929] 1998).

9. Une langue flexionnelle est une langue dans laquelle les lemmes changent de forme selon leur rapport grammatical aux autres mots, dans la phrase. C'est aussi le cas du grec ancien où ces particules ne peuvent être traduites en français que par périphrase ou par omission.

10. Il s'agit ici d'un tapis au motif floral.

11. Cet adjectif-épithète vient du substantif bogatyr', un héros des contes et bylines slaves qui réalise des prouesses à caractère patriotique et militaire. Ce mot désigne par extension une personne qui incarne la force physique, la bravoure et le courage. On dit « la taille de bogatyr' » (très grand). 
12. Dus au système flexionnel et dérivationnel hautement développé de la langue russe, les diminutifs à valeur affective atténuent le discours, lui communiquant tendresse et affect.

13. Le cynisme du tonnelier prend le dessus sur ses sentiments de père lorsqu'il séquestre sa fille dans sa chambre à la fin du roman, après qu'Eugénie lui a avoué avoir donné tous ses louis d'or à son cousin Charles.

14. Prenant ses racines dans le slavon d'église, l'inversion fait le propre des chants psalmodiques orthodoxes, puis, à partir du XIX ${ }^{\mathrm{e}}$ siècle, des textes poétiques.

15. La douceur (krotost') est, dans la religion orthodoxe, l'une des principales qualités d'un homme chrétien : par conséquent, elle fera le socle de la pensée orthodoxe de Dostoïevski ainsi que du sentiment religieux dans ses œuvres.

16. Dans Crime et châtiment, Sonia Marmeladova est décrite par le romancier comme une femme "timide, [à la voix] si douce... ", qui «éprouvait une certaine confusion et une grande honte mêlée à une certaine douceur ». Lorsqu'elle suivra Raskolnikov en Sibérie, les galériens verront en elle l'image de la Vierge : "Chère Sophia Semionovna, tu es notre mère douce et secourable » (Dostoïevski [1866] 1983 : 608).

17. Publiée en novembre 1878 dans le Journal d'un écrivain, La Douce est l'avant-dernière œuvre du romancier russe ; il y sera question du suicide d'une jeune femme de dix-huit ans qui, terrorisée par son époux, se défenestre un an après leur mariage.

18. Les critiques verront dans l'écriture romanesque du romancier russe un mélange complexe de genres et d'influences littéraires, dont le réalisme, le baroque (Tučina), la tragédie (Evnin), le carnavalesque (Bakhtine), voire le symbolisme (Berdjaev).

19. Jacques Derrida dit à ce propos : «Il est cela même que la traduction laisse tomber le corps. Laisser, telle est même l'énergie essentielle de la traduction » $(1967: 312)$.

\section{RÉSUMÉS}

Parue en janvier 1844, alors que Dostoïevski n'est pas encore écrivain, la première traduction en russe d'Eugénie Grandet d'Honoré de Balzac se présente comme un essai où l'acte de traduire se mélange avec celui d'écrire. Ce premier travail rémunéré du futur romancier russe le révèle d'emblée en tant qu'artiste en quête d'indépendance poétique qui réfléchit, au travers de sa traduction, à l'essence même du métier romanesque et au chemin qu'il a à prendre dans le monde des Lettres. Le traducteur laisse libre cours à son imagination; il conteste le mode narratif du roman original, tout en insufflant à la version russe d'Eugénie Grandet un nouvel idéal esthétique tourné vers un mélange complexe de styles dont le réalisme, le romantisme, le tragique et le baroque. La version de Fiodor Dostoïevski est de toute évidence plus qu'une simple traduction: considérée comme une création artistique, elle marque le début de la carrière littéraire de son traducteur, et semble même participer au succès ultérieur de ses écrits.

Published in January 1844, while Dostoevsky was not yet a famous writer, the first translation of Balzac's Eugénie Grandet into Russian presents itself as an essay where the act of translating is interwoven with that of writing. This first translation by the future Russian novelist reveals his quest for poetic independence. Through his translation, Dostoevsky reflects on the very essence of writing as a professional activity and ponders the path that he was to take in the world of letters. The translator gives free rein to his imagination, he challenges the narrative mode of the original novel, while infusing the Russian version of Eugenie Grandet with a new aesthetic ideal 
encompassing different literary styles including realism, romanticism, tragedy and baroque. Fyodor Dostoevsky's version is definitely much more than just a translation: considered as an artistic creation, it marks the beginning of his literary career, and seems to even contribute to the subsequent success of his future writings.

INDEX

Mots-clés : traduction, Balzac (Honoré de), Dostoïevski (Fiodor), Eugénie Grandet, traduction productive, intertextualité

Keywords : translation, Balzac (Honoré de), Dostoevsky (Fyodor), Eugénie Grandet, productive translation, intertextuality

\section{AUTEUR}

\section{LILIA ANDROSENKO}

Université de Franche-Comté, CRIT (EA 3224) 